\title{
Smart Grid Operational functions and Control Challenges by Implementing SSSC Tailored to Optimize performance in between Qatar and KSA on the GCC Electrical-power grid
}

\author{
Tariq Masood ${ }^{* 1}$, Muhammad Tajammal ${ }^{2}$, Samer Karim Shah ${ }^{3}$, Ghulam Hashmi ${ }^{4}$, Suhail Aftab Qureshi ${ }^{5}$, D. P Kothari ${ }^{6}$ \\ ${ }^{1}$ Asset Integrity, Department Qatar Petroleum, Dukhan Operations, Qatar \\ ${ }^{2}$ University of Hail, Hail, Kingdom of Saudi Arabia \\ ${ }^{3}$ Maintenance Department, QAFAC, Doha Qatar \\ ${ }^{4}$ Power System Engineering Department, Saudi Aramco, Dhahran, KSA \\ ${ }^{5}$ University of Engineering and Technology, Lahore Pakistan \\ ${ }^{6} \mathrm{~J}$. D. College of Engineering \& Management, Nagpur India
}

\begin{tabular}{l} 
A R T I C L E I N F O \\
\hline Article history: \\
Received: 19 August, 2017 \\
Accepted: 06 September, 2017 \\
Online: 30 September, 2017
\end{tabular}

Keywords:

Reactive voltage

Voltage regulator

Power flow

\begin{abstract}
A B S T R A C T
This research work is novel technique to control and optimize SSSC (Subsynchronous Series Controller) functions with degree of precision in between Qatar and Kingdom of Saudi Arabia. The SSSC model developed and simulated in order to identify and determine its control and functioning parameters by introducing new tuning parameters based on that the SSSC can be adjusted stringently to witness desired results lead to address outstanding reactive power management issue. The proposed new parameters are contributing significantly to control SSSC functions in multiple directions in a power system network in between QATAR and Kingdom of Saudi Arabia at different time-based transmission contingencies on the GCC Electrical-power grid. Strategically, the SSSC capacity and capability can be utilized fully in between Qatar and Kingdom of Saudi Arabia by introducing and optimizing its control and tuning parameters more tangibly under both steady and dynamic states
\end{abstract}

\section{Introduction}

The SSSC has operational controllability results are clearly indicating that introduction of SSSC in between Qatar and Kingdom of Saudi Arabia power network will equitably improve the power system loadability, curtailing the losses and valueadded sustainability of the power system enactment by addressing control and operational issue throughout the GCC Electricalpower grid.

Hereafter, new SSSC optimization technique can thus be magnificently expended for this type of power system process optimization. This work published in $13^{\text {th }}$ the IET International

*Corresponding Author: Tariq Masood, Asset Integrity, Department Qatar Petroleum, Dukhan Operations, Qatar | Email: t.masood.dr@bath.edu
AC/DC Conference held in Manchester, February 14-16, 2017. Whereas a SSSC determines and validates the three control and effective limits which have been made-to-order at (minimum $(+/-$ ) medium (+/-), and maximum (+/-) compensation. These rheostats functioning limits are regulated by constituting their consequent PI control-values by exercising D.J. Cooper PID regulator Performance to achieve the following [1].

(1) Augmented Power flow

(2) Developed consistency \& controllability

(3) Augmented angle and voltage constancy.

Therefore, FACTS Controllers are convincing candidate technology options centered on that following benefits can be perceived. 


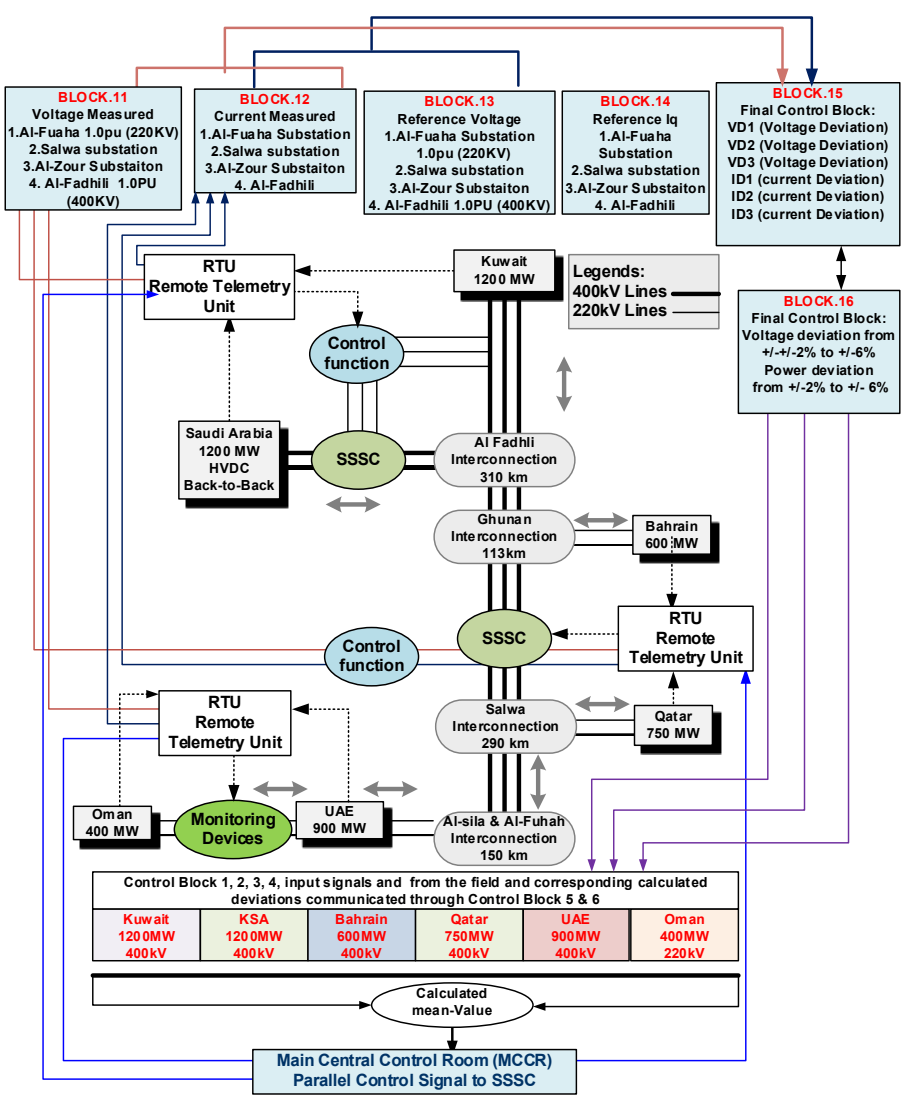

Figure 1. SSSC structure in between Qatar and Kingdom of Saudi Arabia

Figure 1 shows the GCC power network by retaining SSSC at augmented whereabouts by exercising Wideband-DelphiTechnique.

- Model reveals how much notional concentrated power can be dispensed in between QATAR and Kingdom of Saudi Arabia.

- At maximum conjectural power, what is borderline voltage

- Model reveals how much reactance must be retained by the orthodox series capacitance to double the power conveyance in between Qatar and Kingdom of Saudi Arabia if vital and prerequisite.

- Compute the introduced voltage by the series capacitor in between QATAR and Kingdom of Saudi Arabia

- By SSSC how much introduced and instilled/injected reactive voltage $\mathrm{Vr}$ is upheld at diverse compensation of SSSC in between Qatar and Kingdom of Saudi Arabia and Compute the determined power supplied at persistent introduced voltage $\mathrm{Vr}$

- If the load angle decreases from 71 to 0 how much power will be produced by SSSC in between Qatar and Kingdom of Saudi Arabia.

\section{GCC Electrical-power grid background}

The GCC power-grid is constituted and employed into eight premeditated power system operational directions in order to meet national and industrial customers necessities in a consistent and viable demeanors at the GCC Electrical-power grid-network as shown in Figure 1 as considered and described with facts and figures, this is also published in [1] .

www.astesj.com

\section{SSSC Operational Analyses}

Principally, the SSSC-series counterbalance device location is not very much perilous it can be employed anyplace on the GCC Electrical-power grid. In this case the SSSC has been retained at the borderline to decrease the power-line transmission impedance unnaturally by SSSC which is controlling to upsurge the power flow in the power transmission system in (5) and (6) determine how much line impedance $\left(\mathrm{X}_{\mathrm{C}}\right)$ has been Controlled and Managed after employing series but stable capacitor's $\mathrm{K}_{\text {series }}$ factor.

SSSC encompasses of capacitors and reactors to diverge the power-line impedance on domineering need basis vigorously. Predominantly, the SSSC injects the voltage in a series into the power transmission network at the midpoint of Kingdom of Saudi Arabia and Qatar whereas instilled/injected the voltage at midpoint $\mathrm{Vr}$ has been computed in (7). The SSSC stipulates or expends reactive-power in capacitive/inductive control-mode of functions on the GCC power-grid, wherein the SSSC instilled/injected voltage in phase quadrature with the line current of the power network.

Figure 2 denotes maroon trend in the graphical presentation of the SSSC processes in capacitive and inductive mode of functions. Practically, blue trends denote how much real power transferred can transferred when electrical-power transmission network compensation carried-out capacitive, inductive mode of operations or neutral without any compensation factor. Figure 3 signifies that a SSSC has a main and great influence on power steadiness and load flow, but ithas limited impact on voltage profile enhancement as exhibited in (28) that $\mathrm{V}_{\mathrm{m}}, \mathrm{V}_{\mathrm{s}}, \mathrm{V}_{\mathrm{r}}$ has negligible or no effect with SSSC [2].

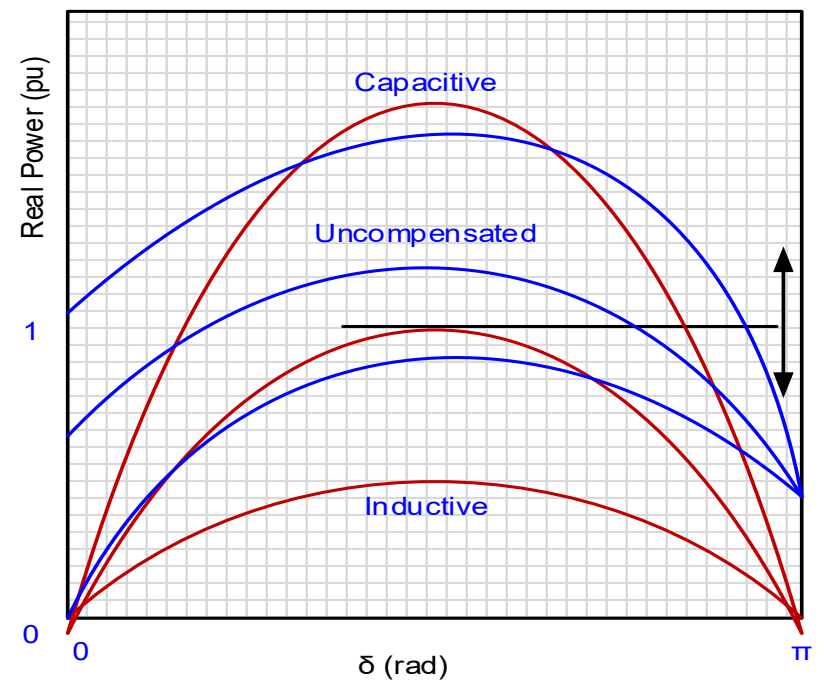

Figure 2. Signify the compensation factor by SSSC [2]

\begin{tabular}{|c|c|c|c|c|}
\hline \multirow{2}{*}{$\begin{array}{c}\text { Principle of Operating } \\
\text { parameters }\end{array}$} & \multirow[b]{2}{*}{ sssc Controller } & \multicolumn{3}{|c|}{ Impact in between Qatar and KSA } \\
\hline & & Load Flow & Stability & Voltage Quality \\
\hline \multirow{3}{*}{$\begin{array}{l}\text { Series Compensation: } \\
\text { To Vary the power } \\
\text { transmission line } \\
\text { impedance }\end{array}$} & $\begin{array}{l}\text { FSC: Fixed Series } \\
\text { Compensation }\end{array}$ & 0 & 000 & 0 \\
\hline & $\begin{array}{l}\text { TPSC: Thryrister protected } \\
\text { Series Compensation }\end{array}$ & 0 & o०o & 0 \\
\hline & $\begin{array}{l}\text { TCSG: Thryrister controlled } \\
\text { Series Compensation }\end{array}$ & oo & 000 & 0 \\
\hline Benchmark & O: Low or no Impact & \multicolumn{2}{|c|}{ O O: Medium } & $\circ \bigcirc 0$ : Strong \\
\hline
\end{tabular}

Figure 3. Denotes the SSSC impact. 


\subsection{By employing Series Fixed Capacitor to upsurge the Power on the GCC Electrical-power grid}

Herein determined conjectural power can be dispensed and distributed without conservative or non-conventional series compensation, the voltage structured transitorily but not vigorously and unvaryingly which is outfitted at $800 \mathrm{~km}$ long electrical-power transmission network from Kingdom of Saudi Arabia to Qatar or vice versa along with its attendant substations. Now if series capacitor is connected at midpoint voltage as stated in Figure 3 to resource and expend sufficient reactive power to counterbalance as prerequisite. Equation (5) expended to compute series compensation factor to convalesce the power system steadiness and double the power at power transmission lines reactance $X_{C}=136.626$ Ohms as intended in (6) and (7) [3].

$K_{\text {series }}=\frac{X_{c}}{2 \times Z_{a}} \cos \frac{\theta}{2}$

$X_{c}=2 \times K_{\text {series }} \times Z_{a} \tan \frac{\theta}{2}=136.626$ Ohms

$P_{\max }=\frac{V^{2}}{Z_{a}-X_{C} \times \operatorname{Sin}\left(43.26^{\circ}\right)}=1223 \mathrm{MW}$

Equation (7) reveals the concentrated power by adapting power transmission line reactance by series capacitor Units.

\subsection{By implementing Series-Capacitor to upsurge the Power on the GCC Electrical-power grid}

The SSSC has a tremendous competence to upturn the electrical Power flow and improve dynamic steadiness to supplant usual and uneconomical fixed series capacitors are expended to govern the prerequisite reactive current at midpoint in between Qatar and Kingdom of Saudi Arabia for recompense [4].

Instill/Injected current with series capacitor is computed by exercising in (8), (9).

$I_{m}=\frac{V \sin \frac{\delta}{2}}{\left(1-K_{\text {series }}\right) Z_{a} \sin \frac{\theta}{2}}=2.1159 p u$

Instilled/injected voltage as given below

$$
\widehat{V}_{r}=1.5237 \times \tan \frac{\theta}{2}=0.8881 p u
$$

Equation (9) reveals the in a Phase injected/instill voltage

$$
V_{r}=\widehat{V}_{r} \times \frac{V}{\sqrt{3}}=205.03 \mathrm{kV}
$$

Equation (10) reveals the instilled/injected voltage in between QATAR and Kingdom of Saudi Arabia. Herein determined power flow can be sustained with unremitting voltage inserted by the SSSC at midpoint. Firstly, the SIL computed and determined by using Equation (11)
$\hat{P}=\frac{P}{P_{n}}=A \cos \delta+B \sin \frac{\delta}{2}$

amount of real power can be dispensed in line with $\mathrm{P}_{\mathrm{n}}$, this is identified as a SIL in contradiction of the real electrical-power delivery.

Where

$A=\frac{1}{\sin \theta}=1.40115 \quad ; \quad B=\frac{\widehat{V_{r}}}{2 \sin \frac{\theta}{2}}=1.14735$

Equation (13) reveals electrical Power flow with unremitting reactive voltage instilled/injected whereas the $\widehat{P}$ is determined

when $\frac{d \hat{P}}{d \delta}=0$

$\frac{d \hat{P}}{d \delta}=A \cos \delta-B \sin \frac{\delta}{2}$

Equation (14) resultant after substituting $\frac{d \hat{P}}{d \delta}=0$ value

$0=A \cos \delta-B \sin \frac{\delta}{2}$

Unassumingly $\cos \delta$ and $\sin \delta$ are factorized as resultant in (15)

$\cos \delta=\cos \left(\frac{\delta}{2}+\frac{\delta}{2}\right) ; \sin \delta=\sin \left(\frac{\delta}{2}+\frac{\delta}{2}\right)$

Where

$\cos (A+B)=\operatorname{Cos} A \cdot \operatorname{Cos} B-\operatorname{Sin} A \cdot \operatorname{Sin} B$

Equation (17) resultant after replacing $\cos \delta$ and $\sin \delta$ values in (16)

$\cos \left(\frac{\delta}{2}+\frac{\delta}{2}\right)=\cos \frac{\delta}{2} \cdot \cos \frac{\delta}{2}-\sin \frac{\delta}{2} \cdot \sin \frac{\delta}{2}$

Equation (17) is the streamlined and resultant in (18)

$\cos \delta=\cos ^{2} \frac{\delta}{2}-\sin ^{2} \frac{\delta}{2}$

Equation (18) $\cos \delta$ value subtract in (14) and resultant from (19)

$0=A\left(\cos ^{2} \frac{\delta}{2}-\sin ^{2} \frac{\delta}{2}\right)-B \sin \frac{\delta}{2}$

Whereas

$\cos ^{2} \frac{\delta}{2}=\left(1-\sin ^{2} \frac{\delta}{2}\right)$

Equation (20) $\cos ^{2} \frac{\delta}{2}$ subtracted in (19) and developed new (21)

$0=A\left(\left(1-\sin ^{2} \frac{\delta}{2}\right)-\sin ^{2} \frac{\delta}{2}\right)-B \sin \frac{\delta}{2}$

By adding the $x=\sin \frac{\delta}{2}$ 
$A\left(1-x^{2}-x^{2}\right)-B x=0$

$-2 A x^{2}+A-B x=0$

$-\left(2 A x^{2}-A+B x\right)=0$

$2 A x^{2}+B x-A=0$

Equation resultant from (25) to convert into Quadratic form.

$\int(x)=\left(x+\frac{b}{2 a}\right)^{2}+\frac{4 a c-b^{2}}{4 a}$

Equation (26) expressed the Quadratic function as detailed below with factors.

$\left(x+\frac{B}{4 A}\right)^{2}+\frac{(4 \times 2 A \times-A)-B^{2}}{4 A}=0$

$\left(x+\frac{B}{4 A}\right)^{2}-\frac{8 A^{2}+B^{2}}{4 A}=0$

Equation (27) and (28) Quadratic roles are defined and extracted and resultant in (29)

$x=-\frac{B}{4 A} \pm \frac{\sqrt{8 A^{2}+B^{2}}}{4 A}$

Equation (29) subtracted A and B factors values as defined and calculated in (12) and resultant in new (30)

$x$

$=-\frac{1.401}{4 \times 1.4735} \pm \frac{\sqrt{8 \times 1.4735^{2}+1.401^{2}}}{4 \times 1.4735}$

Finally, the " $x$ " values are signified as given below:

$x=0.5314=\sin \frac{\delta}{2}=\rightarrow \delta=64.2^{\circ}$

Equation (31) reveals the $x=0.5314$ and electrical power angle resultant

Where

$\hat{P}_{\text {max }}=A \sin \delta+B \cos \theta$

$\hat{P}_{\text {max }}=A \sin \left(64.2^{\circ}\right)+B \cos \left(45.5^{\circ}\right) \hat{P}_{\max }=2.06519$

Equation (32) subtract the A, B, $\varnothing$ and $\delta$ values and resultant the $\hat{P}_{\max }$ value.

$P_{\text {max }}=\hat{P}_{\text {max }} \times \frac{400^{2}}{316.228}=1044.9 \mathrm{MW}$

Equation (34) signifies the concentrated electrical power dispensed and distributed in the GCC Electrical-power grid by retaining SSSC to uphold persistent $\mathrm{Vr}$ vigorously in the Midpoint of QATAR and Kingdom of Saudi Arabia as illustrated in Figure $4[5]$.

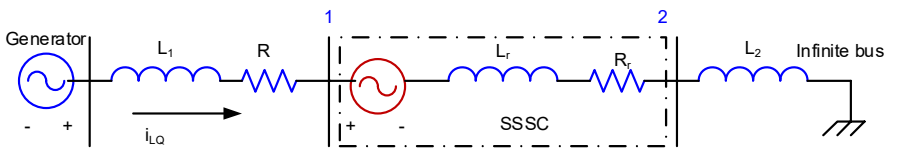

Figure 4: SSSC connected in between Qatar and Kingdom of Saudi Arabia Vm

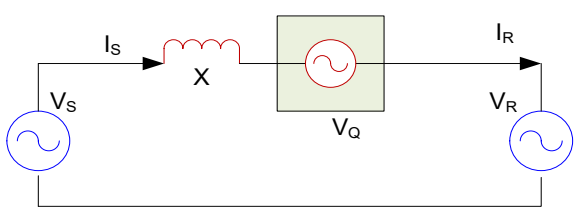

Figure 5: SSSC connected in between Qatar and Kingdom of Saudi Arabia

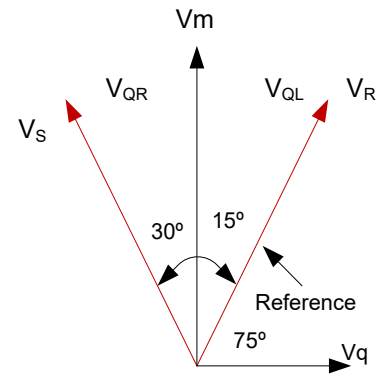

Figure 6. Phasor diagram of voltage profile

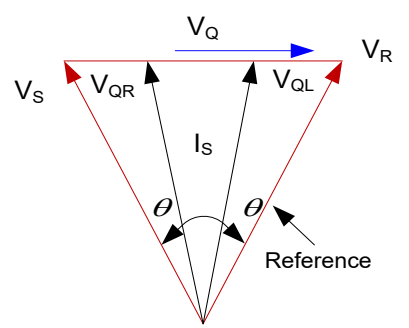

Figure 7. SSSC connected at the midpoint and instilled/injected $V_{Q}$ toward $V_{R}$

Figure 5: signifies that counterbalance device employed in the middle of the of the power transmission line, this is voltage source only dispense reactive electrical power not a real power. Figure 6 displays the influence of location if counterbalance device employment on voltage profile, the voltage on left side of the counterbalance device would be $\mathrm{V}_{\mathrm{QL}}$ and right side $\mathrm{V}_{\mathrm{QR}}$. Figure 7 illustrates that the series counterbalance device employed in the middle the worst voltage-profile occurred at each side of the counterbalance device, whereas the voltage-profile vector aligns with the current vector [5]. Therefore, the midpoint voltage-profile is the same with/without SSSC functions as exhibited in (37) and (38) SSSC power flow with $\delta=0[6]$.

$$
P_{\max }=1.147 \times \frac{400^{2}}{316.2}=580 \mathrm{MW}
$$

$\widehat{V}_{m}=\frac{\cos \frac{\delta}{2}}{\cos \frac{\theta}{2}} \quad \delta=0$

Equation (35) reveals the midpoint voltage-profile if $\delta=0$ and resultant midpoint voltage denotes from (36) 
$\widehat{V}_{m}=\frac{1}{\cos \times 22.76^{\circ}}=1.1 p u$

Equation (35) reveals the midpoint voltage if $\delta=0$ therefore, $V_{S}$ and $V_{R}$ voltage-profile are computed in (37) and (38). Sending end from the Kingdom of Saudi Arabia and receiving end at QATAR

$$
\begin{aligned}
& V_{s}=V_{m} \angle 0-j \frac{V_{r}}{2}=1.171 \angle 0-j \frac{0.8881}{2} \\
& V_{R}=V_{m} \angle 0+j \frac{V_{r}}{2}=1.171 \angle 0-j \frac{0.8881}{2}
\end{aligned}
$$

\subsection{SSSC input Data}

Table 1: operating parameters and associated countries

\begin{tabular}{|l|c|}
\hline \multicolumn{1}{|c|}{ Countries } & Power Exchange \\
\hline To/from Qatar to main transmission line & $400 \mathrm{kV}, 50 \mathrm{~Hz}, 750 \mathrm{MW}$ \\
\hline $\begin{array}{l}\text { To/from Kingdom of Saudi Arabia to main } \\
\text { power-transmission line }\end{array}$ & $400 \mathrm{kV}, 50 \mathrm{~Hz}, 600 \mathrm{MW}$ \\
\hline
\end{tabular}

Table 2: Conventional Reactive Power

\begin{tabular}{|l|c|l|}
\hline Existing Power System & Reactive Power & \multicolumn{1}{c|}{ Distance } \\
\hline $\begin{array}{l}\text { VAR Demand at Al Jasra } \\
\text { substation Kuwait }\end{array}$ & $850 \mathrm{MVAR}$ & $\begin{array}{l}\text { Ghunan Substation to Slawa } \\
\text { Substation total distance } \\
288 \mathrm{Km}\end{array}$ \\
\hline $\begin{array}{l}\text { Reactive Power demand at } \\
\text { Al-Al-Zour Substation }\end{array}$ & $500 \mathrm{MVAR}$ & $\begin{array}{l}\text { Slawa Substation total } \\
\text { distance }=97 \mathrm{Km}\end{array}$ \\
\hline
\end{tabular}

Table 3: Multivariable Controller's Configuration

\begin{tabular}{|l|l|l|}
\hline Al-Zour Substation & $\begin{array}{l}\text { Iq regular: Kp 14dB; } \\
\text { Ki: } 0.014 \mathrm{~dB}\end{array}$ & $\begin{array}{l}\text { Counterbalanced and } \\
\text { regulated voltage at } 0-2 \%\end{array}$ \\
\hline $\begin{array}{l}\text { Rated SSC: }+/-500 \\
\text { MVAR }\end{array}$ & $\begin{array}{l}\text { Ref V: } 1.0 \mathrm{pu} \\
(400 \mathrm{kV})\end{array}$ & $\begin{array}{l}\text { (VC voltage change from } \\
2 \% \text { to } 4 \%)\end{array}$ \\
& & \\
\hline P (proportional): 0.33, & $\begin{array}{l}\text { Droop: } \\
0.033 \mathrm{pu} / 100 \mathrm{MVA} ;\end{array}$ & $\begin{array}{l}\text { (VC voltage change from 4- } \\
\text { Kp: } 14 \mathrm{~dB} ; \mathrm{Ki}:\end{array}$ \\
I (integral) $0.7,0.9,1.2$ & $3500 \mathrm{~dB}$ & \\
\hline
\end{tabular}

\section{Results and Discussion}

\subsection{Maximum level Vr voltage-injection}

It has been simulated and authenticated that further real power can be up-surged in the power transmission line to touch its design specification by up-surging the SSSC compensation factor from $50 \%$ to $65 \%$ reactive voltage instilled/injected voltage would be $0.8864 \mathrm{pu}$ which is equivalent to $204 \mathrm{kV}$ and current Im $=2.1 \mathrm{pu}$ and $\boldsymbol{\delta}=63.87^{\circ}$ will activate consequently at different functioning condition total $1200 \mathrm{MW}$ power will be produced and distributed on the GCC electrical-Electrical-power grid. This is known as a determined compensation by implementing a SSSC and its compensation factor from $50 \%$ to $65 \%$ to obtained projected power produced and delivered in the electrical power transmission network on the GCC electrical-Electrical-power grid.

\subsection{Medium level $\mathrm{Vr}$ voltage-injection}

As simulated by Matlab/SIMULINK and scientific/ mathematical model $\mathrm{V}_{\mathrm{r}}$ instilled/injected voltage $0.5936 \mathrm{pu}$ which is uniform and match to $137 \mathrm{kV}$ at $50 \%$ SSSC reactive voltage compensation whereas the midpoint current $\mathrm{I}_{\mathrm{m}}=1.4459 \mathrm{pu}$, and $\boldsymbol{\delta}=\mathbf{7 1 . 3 2 ^ { \circ }}$ will activate consequently in this functioning circumstance to a total $1079 \mathrm{MW}$ power which can be produced and distributed on the GCC Electrical-power grid as validated by the results. Infact, QATAR and Kingdom of Saudi Arabia system has been devised to produce and distribute concentrated 1200MW electrical-power in the GCC Electrical-power grid during a peak load, therefore Margin is obtainable to upsurge the power up to its projected specification. This is also identified as a medium compensation by a SSSC compensation factor from $20 \%$ to $50 \%$. Therefore, the Control function block expended to readjust PI controller response as exhibited in Figure 10 to diverge the firing angle of the Thyrister control [7].

\subsection{Minimum level Vr voltage-Injection}

In the third challenge of operations, further this model was simulated and authenticated by exercising SSSC Controller. At minimum compensation of the SSSC compensation real power reduce considerably in the power transmission line to touch its projected specification by decreasing the SSSC compensation factor from $5 \%$ to $20 \%$ reactive voltage instilled/injected voltage would be $0.3710 \mathrm{pu}$ which is equal to $86.67 \mathrm{kV}$ and current $\mathrm{Im}=$ $0.974 \mathrm{pu}$ and $\boldsymbol{\delta}=\mathbf{7 7 . 5 7 ^ { \circ }}$ will activate consequently in this functioning condition total $986 \mathrm{MW}$ power will be produced and distributed on the GCC Electrical-power grid. This is identified as a minutest compensation by using a SSSC Controller and its compensation factor from 5 to $20 \%$ to achieve projected electrical power delivery of transmission network on the GCC Electricalpower grid. As instilled/injected voltage up-surged based on compensation from $5 \%$ to $20 \%$ there is considerable power flow and steadiness enhancement but very insignificant influence on voltage-profile as verified simulated consequences in Figure 8 (a) reactive power injection-waveform 8(b) indicates the total power delivered in between Qatar and Kingdom of Saudi Arabia, this is also computed in equation (34). The PI controller configured and demonstrated its operational response in Figure 9. Detailed configuration discussed as given in the appendices [8].

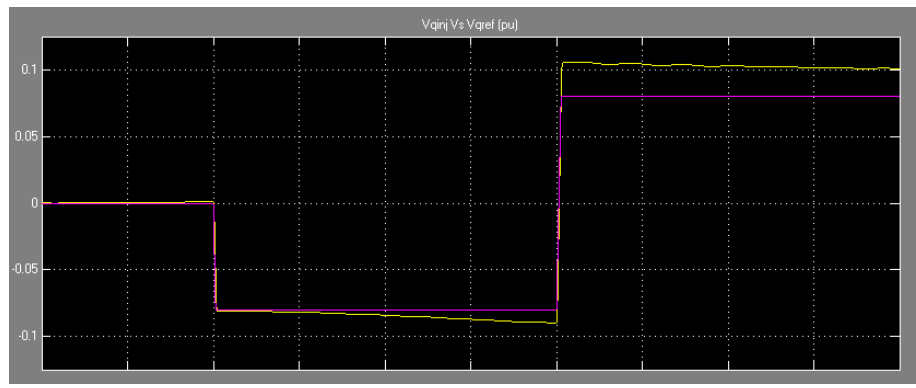

Figure 8 (a). Reactive power injection on the GCC Power Grid

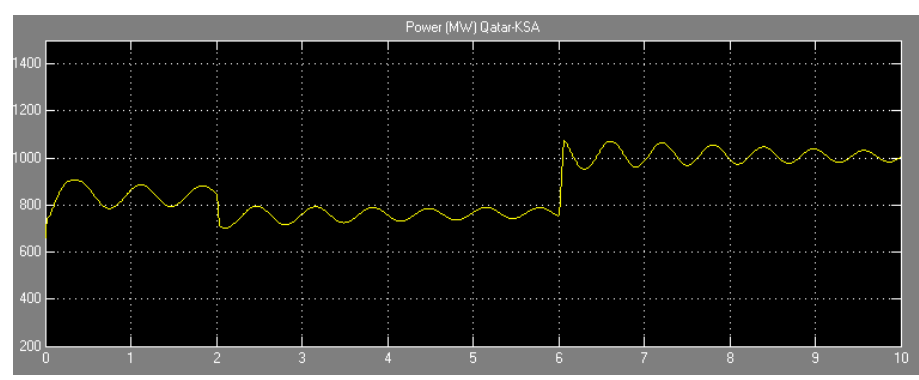

Figure 9 (b). Total active power delivered on the GCC Electrical-power grid 


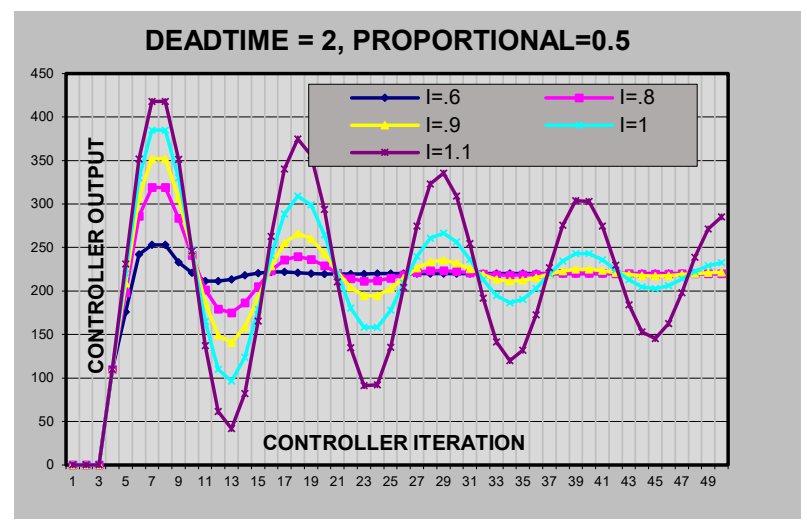

Figure 10. demonstrates the PI controller parameters

\section{Conclusion}

Fundamental creativity of the SSSC application in the GCC Electrical-power grid transmission line compensation is the subject of extensive significance. In this study, it has been computed and simulated how much maximum power can be produced and distributed in between QATAR and Kingdom of Saudi Arabia without any process vagueness. It has been reasonable how much reactance must be provided by the series capacitance to double the power supply in between Qatar and Kingdom of Saudi Arabia. It also has been also identified and unwavering amount of reactive voltage to be instilled/injected in between Qatar and Kingdom of Saudi Arabia in order to provide concentrated power delivery on the GCC Electrical-Power grid.

If the load angle as decreased below from 65 to 0 amount of electrical-power will be produced by SSSC in between Qatar and Kingdom of Saudi Arabia. Herein, the GCC electrical-power network will exclusively upsurge the power system loadability, decrease the losses and improve sustainability of the electricalpower system functioning. The results also illustrates that SSSC is multipurpose equipment with stupendous active capability to improve power system stability margin on the GCC Electricalpower grid. Based on these results, SSSC is a very strongcandidate to be instigated at GCC Electrical-power grid. It also demonstrates optimistic influence on neighboring countries' power system functions at the GCC Electrical-power grid.

Table 4 Terms and Abbreviations

\begin{tabular}{|l|l|}
\hline Name & Abbreviations \\
\hline GCC & Gulf Cooperative Council \\
\hline $\mathrm{Ir}$ & Reactive current \\
\hline $\mathrm{Za}$ & Line Impedance \\
\hline $\mathrm{Vm}$ & Midpoint voltage \\
\hline $\mathrm{Kshunt}$ & series compensation factor in the network \\
\hline $\mathrm{Pn}$ & Surge Impedance \\
\hline KSA & Kingdom of Saudi Arabia \\
\hline
\end{tabular}

\section{Conflict of Interest}

No conflict of interest in this paper has been identified.

\section{Acknowledgment}

I extend my special gratitude to all my coauthors in this paper in particularly late Prof. Dr Abdel-ETY Edris (Icon of FACTS
Technology) at Santa Clara University for their unprecedented support and exceptional contribution to make this study a great success. I am also very grateful to my little Doll Princesses Fatima Masood, who kept me busy full time not giving me space during my research on smart control

\section{References.}

[1] Tariq Masood, R.K. Aggarwal, S.A. Qureshi "Novel Control of a SSSC Connecting the Oman and United Arab Emirates in the GCC power Grid Interconnection" published in IJAPE International Journal of Automation and Power Engineering, Volume 2, issue 1 January, 2013.

[2] Tariq Masood, Abdel Aty Edris, Suahil Aftab Qureshi, Muhammad Tajammal, Murtaza Hashmi DP Kothari, Samer Kareem "SSSC tailored to optimization performance in Between United Arab Emirates and Oman on the GCC Power Grid" published on 14-16 February, 2017 in the 13th IET International Conference on $\mathrm{AC}$ and $\mathrm{DC}$ power Transmission Conference.

[3] Mohammed Rasetgar, Mehdi Saradarzeda "An Improved D-SSSC Voltage and Current Load Balancing Control Strategy Under Unbalanced Load published in 25th Iranian IEEE ICEE 2017.

[4] Mohammed Abdul, Mohd Akram, "Power System Stability Enhancement using Static Synchronous Series Compensator" Published in IEEE (SCOPES)-2016

[5] S. Jamali, A. Kazemi, H. Shateri "Voltage Inversion due to Presence of SSSC on Adjacent Lines and Distance Relay Mal-Operation" published in 2008 IEEE Conference.

[6] D. Rai, S.O. Faried, "Impact of Imbalanced Phase Operation of SSSC on Damping Subsynchronous Resonance published in 2011 IEEE Conference,

[7] Tariq Masood "Improvement of Voltage and power flow control in the GCC Electrical-power grid by using coordinated FACTS Controllers" Ph.D Thesis, published in 2013 at University of Bath, UK.

[8] Tariq Masood, R.K. Aggarwal, Nasser Al-Eamdi "Enhanced Power Flow/Voltage Control in the GCC Grid by SSSC (SUBSYNCHRONOUS SERIES CONTROLLER) Robustness" published by Lambert in 2013 ISBN: 978-3-659-40995-0.

\section{Appendix No.1 \\ PI Controller response computed}

\begin{tabular}{|c|c|c|c|c|c|}
\hline \multicolumn{2}{|c|}{$\begin{array}{l}\text { Proportional } \\
\text { Integral }\end{array}$} & \multicolumn{4}{|l|}{$\begin{array}{l}0.5 \\
0.8\end{array}$} \\
\hline SP & & 220.0 & & & \\
\hline \multirow{4}{*}{ Iteration } & & & ABSOLUTE & & \\
\hline & PV & ERROR & ERROR & OUTPUT & INTEGRAL \\
\hline & & & & 0.00 & 0.00 \\
\hline & & & & 0.00 & 0.00 \\
\hline 1 & 0.0 & 220.00 & 220.00 & 110.00 & 88.00 \\
\hline 2 & 0.0 & 220.00 & 220.00 & 198.00 & 176.00 \\
\hline 3 & 0.0 & 220.00 & 220.00 & 286.00 & 264.00 \\
\hline 4 & 110.0 & 110.00 & 110.00 & 319.00 & 308.00 \\
\hline 5 & 198.0 & 22.00 & 22.00 & 319.00 & 316.80 \\
\hline 6 & 286.0 & -66.00 & 66.00 & 283.80 & 290.40 \\
\hline 7 & 319.0 & -99.00 & 99.00 & 240.90 & 250.80 \\
\hline 8 & 319.0 & -99.00 & 99.00 & 201.30 & 211.20 \\
\hline 9 & 283.8 & -63.80 & 63.80 & 179.30 & 185.68 \\
\hline 10 & 240.9 & -20.90 & 20.90 & 175.23 & 177.32 \\
\hline 11 & 201.3 & 18.70 & 18.70 & 186.67 & 184.80 \\
\hline 12 & 179.3 & 40.70 & 40.70 & 205.15 & 201.08 \\
\hline
\end{tabular}


T. Masood et al. / Advances in Science, Technology and Engineering Systems Journal Vol. 2, No. 6, 20-27 (2017)

\begin{tabular}{|rrrrrr|}
\hline 13 & 175.2 & 44.77 & 44.77 & 223.47 & 218.99 \\
14 & 186.7 & 33.33 & 33.33 & 235.65 & 232.32 \\
15 & 205.2 & 14.85 & 14.85 & 239.75 & 238.26 \\
16 & 223.5 & -3.46 & 3.46 & 236.53 & 236.87 \\
17 & 235.7 & -15.65 & 15.65 & 229.05 & 230.61 \\
18 & 239.7 & -19.75 & 19.75 & 220.74 & 222.71 \\
19 & 236.5 & -16.53 & 16.53 & 214.45 & 216.10 \\
20 & 229.0 & -9.05 & 9.05 & 211.58 & 212.48 \\
21 & 220.7 & -0.74 & 0.74 & 212.11 & 212.19 \\
22 & 214.5 & 5.55 & 5.55 & 214.96 & 214.41 \\
23 & 211.6 & 8.42 & 8.42 & 218.62 & 217.78 \\
24 & 212.1 & 7.89 & 7.89 & 221.72 & 220.93 \\
25 & 215.0 & 5.04 & 5.04 & 223.45 & 222.95 \\
26 & 218.6 & 1.38 & 1.38 & 223.64 & 223.50 \\
27 & 221.7 & -1.72 & 1.72 & 222.64 & 222.81 \\
28 & 223.4 & -3.45 & 3.45 & 221.09 & 221.43 \\
29 & 223.6 & -3.64 & 3.64 & 219.61 & 219.98 \\
30 & 222.6 & -2.64 & 2.64 & 218.66 & 218.92 \\
31 & 221.1 & -1.09 & 1.09 & 218.38 & 218.49 \\
32 & 219.6 & 0.39 & 0.39 & 218.68 & 218.64 \\
33 & 218.7 & 1.34 & 1.34 & 219.31 & 219.18 \\
34 & 218.4 & 1.62 & 1.62 & 219.99 & 219.83 \\
35 & 218.7 & 1.32 & 1.32 & 220.49 & 220.36 \\
36 & 219.3 & 0.69 & 0.69 & 220.70 & 220.63 \\
\hline & & & & & \\
\hline
\end{tabular}

\begin{tabular}{|rrrrrr|}
\hline 37 & 220.0 & 0.01 & 0.01 & 220.64 & 220.63 \\
38 & 220.5 & -0.49 & 0.49 & 220.39 & 220.44 \\
39 & 220.7 & -0.70 & 0.70 & 220.09 & 220.16 \\
40 & 220.6 & -0.64 & 0.64 & 219.84 & 219.91 \\
41 & 220.4 & -0.39 & 0.39 & 219.71 & 219.75 \\
42 & 220.1 & -0.09 & 0.09 & 219.70 & 219.71 \\
43 & 219.8 & 0.16 & 0.16 & 219.79 & 219.78 \\
44 & 219.7 & 0.29 & 0.29 & 219.92 & 219.89 \\
45 & 219.7 & 0.30 & 0.30 & 220.04 & 220.01 \\
46 & 219.8 & 0.21 & 0.21 & 220.11 & 220.09 \\
47 & 219.9 & 0.08 & 0.08 & 220.13 & 220.13 \\
48 & 220.0 & -0.04 & 0.04 & 220.11 & 220.11 \\
49 & 220.1 & -0.11 & 0.11 & 220.05 & 220.06 \\
50 & 220.1 & -0.13 & 0.13 & 220.00 & 220.01 \\
\hline
\end{tabular}

\section{Appendix No. 2}

\section{SSSC Controller configuration}

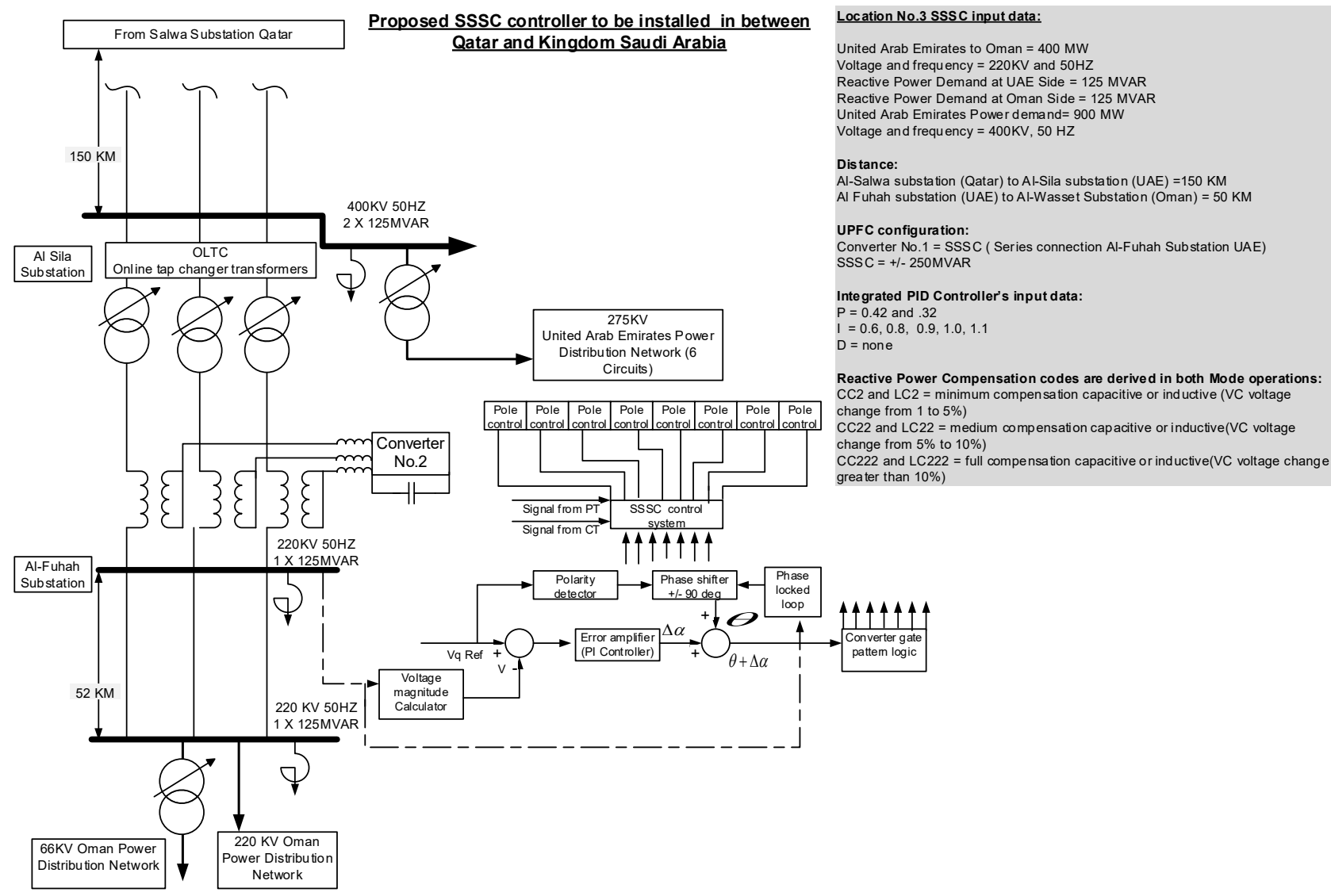


Appendix No. 3

\section{SSSC Centralized and Decentralized Control}
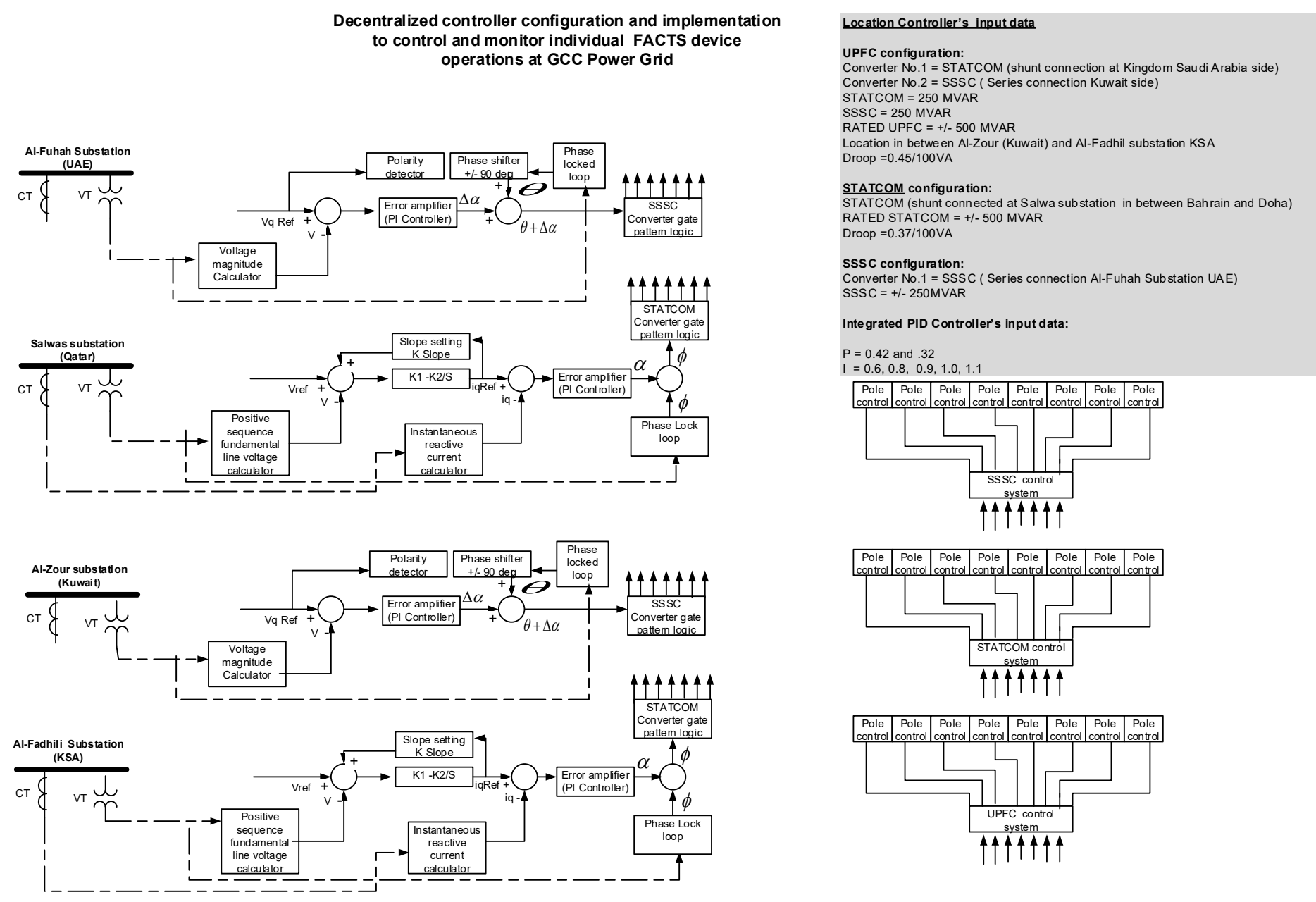